\section{TENDÊNCIA TEMPORAL DAS DOENÇAS RESPIRATÓRIAS NO MUNICÍPIO DE SALVADOR}

\author{
Temporal trend for respiratory diseases in the city of Salvador
}

Artigo Original

\section{RESUMO}

Objetivo: Investigar a distribuição e tendência temporal das principais doenças respiratórias no município de Salvador-BA, Brasil, entre os anos de 1998 e 2007. Métodos: Estudo ecológico de agregado de séries temporais que utilizou a base de dados do DATASUS como fonte de informações para internação hospitalar por causa respiratória. Em seguida, foram construídas taxas padronizadas de morbidade hospitalar para pneumonia, asma e Doença Pulmonar Obstrutiva Crônica (DPOC) segundo sexo e faixa etária para os anos estudados. Resultados: A asma e pneumonia apresentaram maior morbidade entre os indivíduos abaixo de 15 anos e a DPOC entre os indivíduos acima de 59 anos. A taxa de internação por asma reduziu-se de 109,89/100.000 no ano de 1998 para 19,67/100.000 em 2006. Com relação ao sexo os homens apresentaram maiores taxas de internação hospitalar para as três doenças investigadas. As demais doenças respiratórias variaram entre 18,0\% e 37,3\% entre os anos estudados. Conclusões: Os resultados demonstraram tendência temporal de redução das taxas de internação hospitalar por pneumonia, asma e DPOC nos anos de 1998 a 2007; medidas de controle e prevenção constituíram aliados para redução da morbidade dessas doenças.

Descritores: Morbidade; Asma; Pneumonia; Doença Pulmonar Obstrutiva Crônica.

\section{ABSTRACT}

Objective: To investigate the distribution and temporal trend of major respiratory diseases in Salvador, Bahia, Brazil, between 1998 and 2007. Methods: Ecological study of aggregate time series that used the database DATASUS as source of information for hospital admission due to respiratory causes. Then, we constructed standardized rates of hospital admissions for pneumonia, asthma and Chronic Obstructive Pulmonary Disease (COPD) by gender and age group for the years studied. Results: The asthma and pneumonia showed a higher morbidity among individuals below 15 years and COPD among individuals over 59 years. The hospitalization rate for asthma decreased from 109.89 per 100,000 in 1998 to 19.67 per 100,000 in 2006. Regarding gender, men had higher rates of hospitalization for the three diseases studied. Other respiratory diseases ranged between $18.0 \%$ and $37.3 \%$ between the years studied. Conclusions: The results demonstrated trend of reduction on rates of hospital admissions for pneumonia, asthma and COPD in the years 1998 to 2007. Prevention and control measures constituted allies to reduce morbidity of these diseases.

Descriptors: Morbidity; Asthma; Pneumonia; Pulmonary Disease; Chronic Obstructive.

\author{
Jefferson Paixão Cardoso(1) \\ Maria Nice Dutra de Oliveira ${ }^{(1)}$
}

1) Universidade Estadual do Sudoeste da Bahia (UESB) - Jequié (BA) - Brasil
Recebido em: 14/07/2010

Revisado em: $15 / 02 / 2011$ Aceito em: 28/03/2011 


\section{INTRODUÇÃO}

As doenças respiratórias constituem importante causa de morbimortalidade em crianças, adultos jovens e idosos. Considerando as informações de morbidade, estas expressam a doença ou agravos à saúde em determinada população e podem indicar necessidades de reavaliação e expansão dos serviços de saúde e meios para avaliação da situação de saúde da comunidade. No Brasil, a morbidade hospitalar por doença respiratória respondeu por cerca de $13,6 \%$ das internações no ano de 2006. Estes números devem ter influenciado diretamente a demanda dos serviços de saúde, através do aumento pela procura desses serviços com consequentes gastos com internamentos em hospitais e procedimentos em nível ambulatorial na atenção básica ${ }^{(1,2)}$.

As causas responsáveis pelo aparecimento das doenças respiratórias são de origem multifatorial. O fumo e a poluição atmosférica são considerados causas comuns dos eventos mais estudados na literatura que incluem a asma, doença pulmonar obstrutiva crônica (DPOC) e pneumonias. Outros fatores, a exemplo do Índice de Desenvolvimento Humano - IDH, têm sido estudados, procurando avaliar a associação entre estes e o surgimento das doenças respiratórias ${ }^{(3)}$. Entretanto, o fumo é considerado principal poluente responsável pela lesão nas vias respiratórias ${ }^{(4)}$, pois além de prejudicar diretamente quem fuma ${ }^{(5-7)}$, pode ter efeitos negativos à saúde de pessoas que tenham contato indireto (não fumantes ou fumantes passivos).

Estudos realizados na cidade de São Paulo ${ }^{(3,8)}$ demonstraram que os níveis de poluição têm provocado danos à saúde da população. Tem-se evidenciado a associação entre os níveis de poluentes atmosféricos e internações por afecções respiratórias de menores de 15 anos e mortes de idosos $^{(8)}$, recolocando o problema da poluição e estimulando a discussão sobre medidas de prevenção. Dessa forma, a influência dos poluentes atmosféricos sobre o desenvolvimento das doenças respiratórias vem aumentando a preocupação dos pesquisadores e gestores de diversas áreas que não apenas saúde, tais como ambiente e planejamento socioeconômico ${ }^{(3)}$.

No Brasil, a carência de dados epidemiológicos consistentes sobre morbidade respiratória dificulta $\mathrm{o}$ planejamento e a execução de ações efetivas de prevenção e promoção à saúde ${ }^{(9)}$. Contudo, em virtude da importância epidemiológica das doenças respiratórias, faz-se necessário conhecer a distribuição desses eventos, avaliando características segundo pessoa, tempo e espaço. Para isto, o uso das bases de dados sobre morbidade hospitalar disponibilizados pelo Ministério da Saúde, mesmo sofrendo críticas fundamentadas, tem-se constituído fonte de informações com regularidade e acessibilidade. Pensandose nessas possibilidades, o estudo objetivou investigar a distribuição e tendência temporal das principais doenças respiratórias no município de Salvador-BA entre os anos de 1998 e 2007.

\section{MÉTODOS}

Realizou-se um estudo, do tipo ecológico, de agregados de séries temporais com dados de internações hospitalares por local de residência, no município de Salvador-BA, abrangendo o período de 1998 a 2007. Os anos de 2008 e 2009 não foram incluídos, devido à transição dos sistemas para plataforma net.

O município de Salvador, capital do estado da Bahia, fica localizado no litoral do estado e possui área territorial de $707 \mathrm{Km}^{2}$. De acordo com estimativa censitária do Instituto Brasileiro de Geografia e Estatística (IBGE) para o ano de 2007, Salvador possuía população de aproximadamente 2.754.950 habitantes, o que corresponde a 19,56\% da população do estado ${ }^{(10)}$. Segundo informações do Cadastro Nacional de Estabelecimentos de Saúde (CNES) ${ }^{(10)}$, até dezembro de 2007, Salvador possuía 63 hospitais, destes, 4 hospitais estavam sob administração federal, 18 estadual e 41 privado.

As informações sobre as internações hospitalares por doenças respiratórias ocorridas no município foram obtidas a partir dos bancos de dados informatizados, disponibilizados pelo Departamento de Informática do Ministério da Saúde (DATASUS) ${ }^{(10)}$. Estes bancos contêm informações de todas as internações realizadas através das Autorizações de Internação Hospitalar (AIH) do Sistema Único de Saúde (SUS).

Para coleta de dados referente à morbidade hospitalar por doenças respiratórias acessou-se a base de dados através do sistema Tabnet no site do DATASUS (www.datasus.gov. br). Nesse sistema, identificaram-se os campos das variáveis a serem estudadas (sexo, faixa etária, causa da internação por doença respiratória e ano da ocorrência da internação). Em seguida, realizou-se cruzamentos das primeiras segundo os anos de estudo (1998 a 2007). O cruzamento dessas variáveis geraram tabelas que foram transportadas para análise, utilizando o software Tabwin ${ }^{(10)}$. Todos os dados com os campos sexo e faixa etária, considerados ignorados, foram desprezados na análise. Os dados relativos à população do município de Salvador foram obtidos no IBGE (www.ibge.gov.br) para o ano censitário de 2000 e projeções intercensitárias para os anos de 1998 a 1999 e 2001 a 2007.

Construíram-se indicadores de morbidade geral de internação hospitalar por doença respiratória e indicadores segundo a causa da internação hospitalar por doença respiratória, sexo e faixa etária para cada ano estudado. 
Para os indicadores de morbidade geral, dividiu-se o número de internações por causa respiratória ocorridas no ano pela população total do mesmo período. Os indicadores de morbidade por causa de internação, sexo e faixa etária foram elaborados dividindo-se o número de internações pela causa de internação, sexo e faixa etária segundo a população específica a cada grupo. Para comparação dos coeficientes de morbidade geral entre município, estado e país foram construídas taxas padronizadas, utilizando o método direto ${ }^{(11)}$ com referência na população do ano de 2000. A base populacional para taxas padronizadas foi de 10.000 habitantes. As faixas etárias disponibilizadas no sistema Tabnet foram recategorizadas em três faixas etárias: menor que 15 anos, 15 a 59 anos e maior que 59 anos de idade. A base populacional para construção dos indicadores de morbidade segundo causa respiratória, faixa etária e sexo foi de 100.000 habitantes.

A causa da doença respiratória foi definida a partir da décima revisão da Classificação Internacional das Doenças (CID 10). Os dados seguiram o Capítulo X da CID 10, que trata sobre "Doenças do aparelho respiratório", CID J00-J99 ${ }^{(12)}$. Dessa forma, o estudo incluiu os dados que tiveram como causa de internamento as pneumonias, asma e DPOC. Para fins de comparação criou-se a categoria "Demais afecções", que incluiu o restante das doenças respiratórias apresentadas na CID 10 (doenças intersticiais pulmonares, pneumotórax, afecções de vias aéreas superiores, entre outras).

Após a construção dos indicadores de morbidade seguiu-se a elaboração das proporções de internações a partir grupo de causas gerais de internamento hospitalar e entre as causas respiratórias de internamento segundo faixa etária. Em última etapa, foram construídas séries temporais com os indicadores de morbidade segundo variáveis de análise. Este estudo foi aprovado pelo Comitê de Ética de Pesquisa da Universidade Estadual do Sudoeste da Bahia, protocolo n.233/2008.

\section{RESULTADOS}

No período de 1998 a 2007 ocorreram 16.057.457 internações hospitalares causadas por doenças respiratórias em todo o país. Para o mesmo período, 1.542 .851 internação na Bahia e 88.575 no município de Salvador (Tabela I).

Do total de internações por causa respiratória em Salvador, os anos de 1999 e 2000 representaram, respectivamente, $8,3 \%$ e 7,4\%. Observou-se que a ocorrência de morbidade respiratória foi semelhante para os anos estudados. Porém, o ano de 2006 apresentou a menor proporção, com $6,2 \%$ casos (Tabela I).

Quando comparados a taxa geral de morbidade respiratória em Salvador com as taxas estaduais e nacional observou-se, para todos os anos estudados, taxas menores. Entretanto, as taxas para Salvador e Brasil, no ano de 1999, apresentaram valores de 55,92/10.000 e 120,13/10.000, respectivamente. $\mathrm{Na}$ Bahia, o destaque foi para o ano de 2000, com taxa de 149,16/10.000. Contudo, houve decrescimento para os anos subsequentes.

A distribuição das doenças respiratórias segundo faixa etária está discriminada na Tabela II. Observou-se que tanto

Tabela I - Distribuição das doenças respiratórias segundo localidade. Salvador-BA, 1998-2007.

\begin{tabular}{cccccccccc}
\hline Ano & \multicolumn{3}{c}{ Salvador } & \multicolumn{3}{c}{ Bahia } & \multicolumn{3}{c}{ Brasil } \\
\hline & $\mathrm{N}$ & $\%$ & Coef & $\mathrm{N}$ & $\%$ & Coef & $\mathrm{N}$ & $\%$ & Coef \\
\cline { 2 - 9 } 1998 & 10984 & $7,2 \%$ & 48,30 & 166873 & $16,6 \%$ & 129,85 & 1934711 & $16,5 \%$ & 119,58 \\
1999 & 12877 & $8,3 \%$ & 55,92 & 190384 & $18,1 \%$ & 146,53 & 1969462 & $16,5 \%$ & 120,13 \\
2000 & 10921 & $7,4 \%$ & 44,70 & 194956 & $18,6 \%$ & 149,16 & 1936444 & $16,2 \%$ & 114,04 \\
2001 & 8892 & $6,5 \%$ & 35,77 & 178213 & $17,5 \%$ & 134,87 & 1834903 & $15,6 \%$ & 106,44 \\
2002 & 8770 & $6,6 \%$ & 34,79 & 186140 & $18,3 \%$ & 139,71 & 1820633 & $15,5 \%$ & 104,25 \\
2003 & 9977 & $7,4 \%$ & 39,03 & 174085 & $17,0 \%$ & 129,52 & 1746688 & $15,0 \%$ & 98,75 \\
2004 & 10006 & $7,7 \%$ & 38,60 & 168352 & $17,3 \%$ & 124,22 & 1713996 & $14,9 \%$ & 95,70 \\
2005 & 8725 & $6,6 \%$ & 32,63 & 145649 & $16,0 \%$ & 105,43 & 1565743 & $13,7 \%$ & 85,01 \\
2006 & 7976 & $6,2 \%$ & 29,38 & 138199 & $15,6 \%$ & 99,77 & 1541113 & $13,6 \%$ & 82,51 \\
2007 & 8494 & $6,6 \%$ & 30,83 & 146480 & $16,0 \%$ & 104,01 & 1550295 & $13,7 \%$ & 81,88 \\
\hline
\end{tabular}

Taxa de morbidade padronizada por 10.000 habitantes.

Fonte: Ministério da Saúde, Secretaria de Vigilância em Saúde e Departamento de Informática do SUS. 
a pneumonia quanto a asma, na faixa etária menor que 15 anos, apresentaram maiores proporções nos períodos estudados, variando de $78,2 \%$ a $84,9 \%$ para pneumonia e $84,4 \%$ a $91,8 \%$ para a asma. Essas duas doenças sofreram decréscimo acentuado com aumento da faixa etária. Destaca-se, ao longo do período estudado, redução acentuada no número de internações por pneumonias: 5.707 em 1998 para 4.162 internações em 2006. A DPOC apresentou relação inversa, com maior proporção na faixa etária "maior que 59 anos" variando entre 50,4\% e 67,1\%. No período estudado houve aumento percentual atingindo 63,6\% em 2007.

As taxas de morbidade respiratória segundo sexo foram mais expressivas entre os homens para as três doenças respiratórias investigadas. No entanto, a diferença entre as taxas por sexo na DPOC foram mais acentuadas, aproximadamente duas vezes maior entre os homens. No ano de 2001 observou-se situação invertida, maior taxa entre as mulheres com asma, porém, esta diferença não foi expressiva (Tabela III).

Para todos os anos estudados a distribuição percentual apresentou-se semelhante entre o grupo de doenças respiratórias. Porém, a pneumonia foi a principal causa de internamento, seguido da asma e DPOC (Figura 1). A pneumonia representou mais de $50 \%$ das internações para os anos de 1998, 2005, 2006 e 2007. Houve declínio das ocorrências de pneumonia entre os anos de 1998 e 2002. A asma manteve valores estáveis no período de 1998 a 2004, variando entre $26,3 \%$ até $20,1 \%$ do total de internações, mas sofreu queda acentuada nos anos subsequentes, chegando ao valor de 7,7\% no ano de 2006 .

A Figura 2A apresenta a evolução temporal das internações por afecções respiratórias para faixa etária

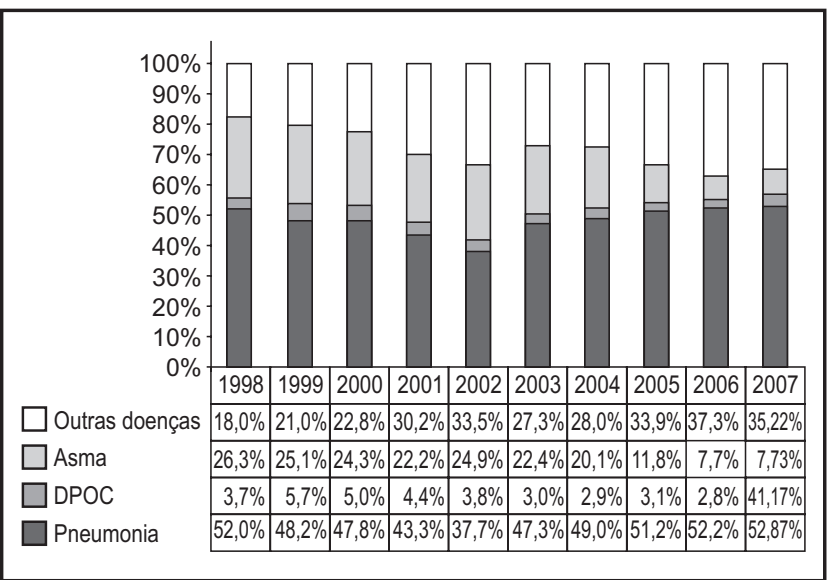

Figura 1 - Distribuição da morbidade respiratória segundo grupos de causa. Salvador-BA, 1998-2007.

Fonte: Ministério da Saúde, Secretaria de Vigilância em Saúde e Departamento de Informática do SUS. menor que 15 anos. Pneumonia e asma mantiveram, ao longo do período, taxas em declínio. Entretanto, a asma apresentou queda mais acentuada das taxas em relação aos demais tipos de afecções respiratórias, variando de 109,89/100.000 no ano de 1998 para 19,67 em 2006, enquanto as taxas para pneumonias variaram de 196,95/ 100.000 no ano de 1998 para 119,89/100.000 em 2006. A DPOC manteve taxas menores, seguindo também tendência temporal de declínio.
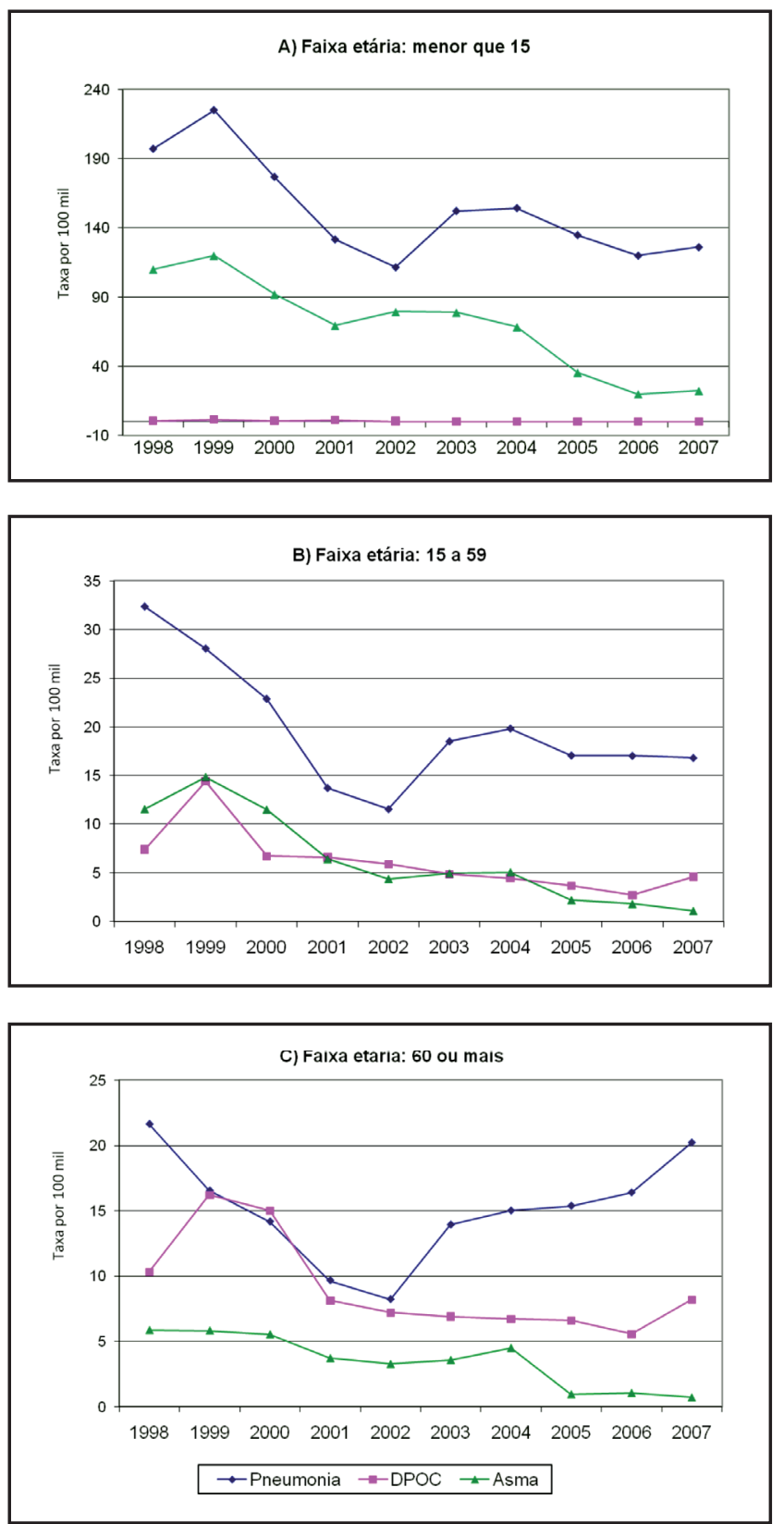

Figura 2 - Evolução temporal das doenças respiratórias segundo doença respiratória e faixa etária. Salvador-BA, 1998-2007.

Taxa de morbidade por 100.000 habitantes.

Fonte: Ministério da Saúde, Secretaria de Vigilância em Saúde e Departamento de Informática do SUS. 
Tabela II - Distribuição da morbidade respiratória segundo faixa etária. Salvador-BA,1998-2007.

\begin{tabular}{|c|c|c|c|c|c|c|c|}
\hline \multirow[b]{2}{*}{1998} & \multicolumn{2}{|c|}{$<15$} & \multicolumn{2}{|c|}{15 a 59} & \multicolumn{2}{|c|}{$>59$} & \multirow[t]{2}{*}{ Total } \\
\hline & $\mathbf{N}$ & $\%$ & $\mathbf{N}$ & $\%$ & $\mathbf{N}$ & $\%$ & \\
\hline Pneumonia & 4479 & 78,5 & 736 & 12,9 & 492 & 8,6 & 5707 \\
\hline$D P O C$ & 8 & 2,0 & 168 & 41,0 & 234 & 57,1 & 410 \\
\hline Asma & 2499 & 86,4 & 262 & 9,1 & 133 & 4,6 & 2894 \\
\hline Outras doenças - 1999 & 840 & 42,6 & 786 & 39,8 & 347 & 17,6 & 1973 \\
\hline Pneumonia & 5179 & 83,5 & 646 & 10,4 & 381 & 6,1 & 6206 \\
\hline$D P O C$ & 36 & 4,9 & 331 & 44,7 & 373 & 50,4 & 740 \\
\hline Asma & 2758 & 85,3 & 341 & 10,5 & 134 & 4,1 & 3233 \\
\hline Outras doenças - 2000 & 1068 & 39,6 & 1029 & 38,1 & 601 & 22,3 & 2698 \\
\hline Pneumonia & 4317 & 82,7 & 559 & 10,7 & 346 & 6,6 & 5222 \\
\hline$D P O C$ & 18 & 3,3 & 163 & 29,7 & 367 & 67,0 & 548 \\
\hline Asma & 2244 & 84,4 & 280 & 10,5 & 135 & 5,1 & 2659 \\
\hline Outras doenças - 2001 & 842 & 33,8 & 1079 & 43,3 & 571 & 22,9 & 2492 \\
\hline Pneumonia & 3271 & 84,9 & 341 & 8,9 & 240 & 6,2 & 3852 \\
\hline$D P O C$ & 22 & 5,7 & 163 & 42,1 & 202 & 52,2 & 387 \\
\hline Asma & 1721 & 87,3 & 159 & 8,1 & 92 & 4,7 & 1972 \\
\hline Outras doenças - 2002 & 759 & 28,3 & 1267 & 47,3 & 655 & 24,4 & 2681 \\
\hline Pneumonia & 2810 & 84,9 & 291 & 8,8 & 207 & 6,3 & 3308 \\
\hline$D P O C$ & 6 & 1,8 & 148 & 44,2 & 181 & 54,0 & 335 \\
\hline Asma & 1996 & 91,2 & 110 & 5,0 & 82 & 3,7 & 2188 \\
\hline Outras doenças - 2003 & 1238 & 42,1 & 1066 & 36,3 & 635 & 21,6 & 2939 \\
\hline Pneumonia & 3888 & 82,4 & 473 & 10,0 & 356 & 7,5 & 4717 \\
\hline$D P O C$ & 3 & 1,0 & 123 & 40,7 & 176 & 58,3 & 302 \\
\hline Asma & 2013 & 90,3 & 126 & 5,7 & 91 & 4,1 & 2230 \\
\hline Outras doenças - 2004 & 1015 & 37,2 & 1016 & 37,2 & 697 & 25,5 & 2728 \\
\hline Pneumonia & 3997 & 81,6 & 514 & 10,5 & 389 & 7,9 & 4900 \\
\hline$D P O C$ & 3 & 1,0 & 115 & 39,4 & 174 & 59,6 & 292 \\
\hline Asma & 1769 & 87,7 & 131 & 6,5 & 116 & 5,8 & 2016 \\
\hline Outras doenças - 2005 & 1129 & 40,4 & 1047 & 37,4 & 622 & 22,2 & 2798 \\
\hline Pneumonia & 3602 & 80,6 & 456 & 10,2 & 411 & 9,2 & 4469 \\
\hline$D P O C$ & 1 & 0,4 & 97 & 35,4 & 176 & 64,2 & 274 \\
\hline Asma & 943 & 91,8 & 59 & 5,7 & 25 & 2,4 & 1027 \\
\hline Outras doenças - 2006 & 1378 & 46,6 & 1107 & 37,5 & 470 & 15,9 & 2955 \\
\hline Pneumonia & 3255 & 78,2 & 462 & 11,1 & 445 & 10,7 & 4162 \\
\hline$D P O C$ & 1 & 0,4 & 73 & 32,4 & 151 & 67,1 & 225 \\
\hline Asma & 534 & 87,4 & 49 & 8,0 & 28 & 4,6 & 611 \\
\hline Outras doenças - 2007 & 1352 & 45,4 & 1121 & 37,6 & 505 & 17,0 & 2978 \\
\hline Pneumonia & 3471 & 77,3 & 463 & 10,3 & 557 & 12,4 & 4491 \\
\hline$D P O C$ & 3 & 0,8 & 126 & 35,6 & 225 & 63,6 & 354 \\
\hline Asma & 608 & 92,5 & 30 & 4,6 & 19 & 2,9 & 657 \\
\hline Outras doenças & 1263 & 42,2 & 1141 & 38,1 & 588 & 19,7 & 2992 \\
\hline
\end{tabular}

Fonte: Ministério da Saúde, Secretaria de Vigilância em Saúde e Departamento de Informática do SUS. 
Tabela III - Distribuição da morbidade respiratória segundo sexo. Salvador-BA, 1998-2007.

\begin{tabular}{lccccccccccc}
\hline & Sexo & $\mathbf{1 9 9 8}$ & $\mathbf{1 9 9 9}$ & $\mathbf{2 0 0 0}$ & $\mathbf{2 0 0 1}$ & $\mathbf{2 0 0 2}$ & $\mathbf{2 0 0 3}$ & $\mathbf{2 0 0 4}$ & $\mathbf{2 0 0 5}$ & $\mathbf{2 0 0 6}$ & $\mathbf{2 0 0 7}$ \\
\hline Pneumonia & $\mathrm{M}$ & 140,23 & 150,86 & 119,60 & 83,40 & 74,75 & 101,47 & 102,19 & 92,20 & 84,09 & 89,61 \\
& $\mathrm{~F}$ & 110,72 & 118,64 & 94,10 & 71,57 & 56,50 & 83,05 & 86,84 & 74,96 & 69,21 & 75,80 \\
DPOC & $\mathrm{M}$ & 11,30 & 21,19 & 13,59 & 10,22 & 8,57 & 7,31 & 7,06 & 6,58 & 11,86 & 8,03 \\
& $\mathrm{~F}$ & 6,73 & 10,94 & 8,84 & 5,35 & 4,72 & 4,50 & 4,20 & 3,67 & 10,64 & 5,01 \\
Asma & $\mathrm{M}$ & 67,15 & 75,26 & 60,58 & 39,47 & 47,93 & 47,25 & 40,12 & 21,32 & 1,44 & 13,63 \\
& $\mathrm{~F}$ & 60,11 & 65,14 & 48,26 & 39,87 & 38,88 & 39,98 & 37,65 & 17,09 & 0,77 & 10,57 \\
Outras doença, & $\mathrm{M}$ & 49,07 & 64,92 & 54,89 & 54,11 & 60,35 & 58,44 & 60,84 & 47,02 & 63,46 & 65,27 \\
& $\mathrm{~F}$ & 37,68 & 52,24 & 47,11 & 53,75 & 56,26 & 48,27 & 47,10 & 47,02 & 109,72 & 44,94 \\
\hline
\end{tabular}

M: Masculino, F: Feminino.

Taxa de morbidade por 100.000 habitantes.

Fonte: Ministério da Saúde, Secretaria de Vigilância em Saúde e Departamento de Informática do SUS

Para indivíduos com faixa etária entre 15 a 59 anos a taxa de morbidade para pneumonia apresentou queda importante entre os anos de 1998 e 2002, apresentou leve ascensão até o ano de 2004 e mantendo níveis estáveis até o ano de 2007, onde constatou-se taxa de morbidade de 16,81/100.000 (Figura 2B). Asma e DPOC mantiveram a tendência de queda de suas taxas, chegando a valores próximos de sete dos nove anos estudados.

Avaliando-se a distribuição da morbidade respiratória segundo causa por ano de estudo em indivíduos maiores de 59 anos (Figura 2C), observou-se redução das taxas de morbidade respiratória por pneumonia entre os anos de 1998 a 2002, seguido por aumento progressivo até o ano de 2007. A taxa de morbidade para DPOC variou de 5,56/100.000 a 16,20/100.000, havendo declínio entre os anos de 1999 e 2006. A asma manteve taxas relativamente estáveis até o ano de 2004. Porém, entre os anos de 2004 e 2005 sofre queda acentuada, passando de 4,47/ $100.000 \mathrm{em} 2004$ para 0,69/100.000 em 2007.

\section{DISCUSSÃO}

O estudo identificou maior número de internações no sexo masculino para as três doenças investigadas. Estudos que analisaram a morbidade hospitalar por causas respiratórias, também observaram que o número de pacientes do sexo masculino hospitalizados foram maiores em relação ao sexo feminino ${ }^{(2,3)}$. Estudo realizado no município de São Paulo encontrou o número de internações por asma ligeiramente superior no sexo feminino ${ }^{(1)}$.

A pneumonia representou a principal causa de internação hospitalar no município de Salvador entre os anos de 1998 e 2007. Estes achados são semelhantes aos encontrados em outros estudos ${ }^{(1,13)}$, entre eles estudo realizado no município de São Paulo demonstrou que 47\% das internações por doenças respiratórias tiveram como causa a pneumonia. As internações por asma e DPOC representaram, respectivamente, $12,5 \%$ e $5,8 \%$ desse total ${ }^{(1)}$.

Observou-se tendência de declínio das internações por pneumonia em pessoas menores que 15 anos nos períodos estudados. Mesmo com pequenas oscilações a tendência foi de queda. Estudo realizado em período anterior demonstrou também tendência de queda da pneumonia na região metropolitana de Salvador ${ }^{(14)}$. O declínio das taxas de internação hospitalar pode ter sido resultado do atendimento de casos de pneumonia na atenção básica, em nível ambulatorial e maior atenção aos fatores de risco como aglomeração domiciliar, tabagismo, instrução da mãe, poluição ambiental, peso ao nascer da criança, desnutrição e desmame precoce ${ }^{(1)}$. A redução da morbidade hospitalar na região Nordeste acompanhou a ampliação da cobertura do SUS, consequentemente aumento da oferta aos serviços de saúde ${ }^{(14)}$. Dados para o Brasil, no ano 2002, revelaram que a pneumonia foi responsável por $14,2 \%$ dos óbitos e por $36,3 \%$ dos pacientes internados por causas respiratórias ${ }^{(10)}$. Os achados para o município de Salvador foram semelhantes em relação aos dados nacionais no mesmo período.

A causa de morte por pneumonia pode ser evitável, a partir da instituição de medidas simples, acessíveis, de baixo custo e que não exigem tecnologia diagnóstica, assim como recursos terapêuticos sofisticados na maioria das vezes. $\mathrm{O}$ atraso ou erro no diagnóstico e na terapêutica podem constituir causas potenciais nos casos de óbito por pneumonia ${ }^{(15)}$.

No presente estudo, a asma apresentou, entre os anos estudados, taxas bem maiores na faixa etária de menores de 15 anos, corroborando com outras pesquisas que encontraram $26,5 \%$ em crianças residentes no município 
do Rio de Janeiro e pico ${ }^{(16)} 7,5 \%$ nos Estados Unidos ${ }^{(17)}$. Mesmo sendo considerada causa principal de adoecimento na infância, esse evento pode ser evitado quando a pessoa com asma e familiares são adequadamente orientados sobre a doença e têm acesso aos cuidados médicos adequados ${ }^{(17)}$. Tais cuidados podem inclusive evitar problemas respiratórios relacionados à infância, em adolescentes e também adultos jovens ${ }^{(18)}$.

Estudos que investigaram fatores de risco para hospitalizações na asma observaram que intervenções efetivas, incluído prevenção e controle podem reduzir, de maneira eficaz, esses indicadores ${ }^{(19,20)}$. No município de Salvador, o declínio acentuado das taxas de morbidade por asma entre as pessoas na faixa etária menor que quinze anos podem ter sido influenciado pela aderência das pessoas ao tratamento dessa patologia ${ }^{(21)}$. Programas de controle da asma no município teriam contribuído para redução dessas $\operatorname{taxas}^{(22,23)}$.

Neste estudo não se observou diferenças expressivas entre os sexos. Porém, outros achados investigaram a prevalência da asma e sintomas respiratórios segundo idade e gênero ${ }^{(24)}$ e uso de tabaco ${ }^{(25)}$, revelando a necessidade do diagnóstico mais preciso e precoce da asma. Na Costa Rica ${ }^{(24)}$, a ocorrência de sintomas respiratórios (diagnóstico de asma e presença de sibilância) entre os meninos era maior na faixa etária de 6 a 7 anos $(53,5 \%)$ e entre as meninas na faixa etária de 13 a 14 anos $(40,5 \%)$. O estudo realizado em escolares do Distrito Federal ${ }^{(26)}$ revelou prevalência de $12,1 \%$ de asma em crianças na faixa etária de seis a sete anos e $13,8 \%$ na de treze a catorze anos ${ }^{(24)}$.

As taxas de internação por DPOC aumentaram acentuadamente com a idade e foram maiores em indivíduos do sexo masculino. Esta situação, segundo a literatura, tem como principais fatores o tabagismo e a maior exposição ocupacional entre os homens ${ }^{(27,28)}$. Estudo realizado em São Paulo demonstrou taxas maiores de morbidade hospitalar por DPOC em indivíduos do sexo feminino ${ }^{(1)}$. As medidas de controle e conscientização ao consumo do tabaco (principal fator risco para DPOC) podem ter contribuído para redução dessas taxas.

A DPOC é responsável por uma considerável parte dos atendimentos médicos de emergência, das consultas ambulatoriais e das internações hospitalares. A doença causa redução da produtividade no trabalho e da expectativa de vida, aposentadoria precoce por incapacidade física e onera financeiramente o Estado, tanto pelos altos custos relacionados ao atendimento nos serviços de saúde e gastos sociais para as pessoas em idade produtiva (trabalhador

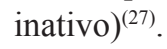

Os resultados obtidos a partir das análises dos dados referiram-se ao grupo da população atendida pelo SUS e não incluiu nesta investigação parcela da população que faz uso das redes conveniada e particular de assistência hospitalar no mesmo período do estudo. Dessa forma, o estudo esteve sujeito ao viés de seleção que poderia gerar sub-representações das doenças investigadas.

É importante lembrar que as taxas de morbidade poderiam incluir internações da mesma pessoa (inclusão no numerador). Por isso, não foi possível estimar com precisão a prevalência ou incidência das doenças em estudo.

Os dados desde estudo poderiam estar supra ou subnotificados. Estas distorções podem ser influenciadas na precisão do diagnóstico na admissão hospitalar do paciente, no preenchimento do formulário e na alimentação no sistema. Estudos realizados para avaliar a confiabilidade dos dados do SIH levantaram várias situações que poderiam levar a registros subnotificados no sistema ${ }^{(29)}$. Porém, outros estudos têm demonstrado grau elevado de concordância para o diagnóstico e grande confiabilidade para as informações demográficas contidas nas AIH's quando comparados com prontuários ${ }^{(30)}$.

Também não foi possível avaliar a influência da poluição ambiental e variação sazonal das doenças estudadas. Alguns estudos têm demonstrado a influência desses fatores para ocorrência dos acometimentos respiratórios em população urbana $^{(1,3,8,24)}$.

\section{CONCLUSÕES}

Este estudo possibilitou investigar a tendência e distribuição temporal da asma, DPOC e pneumonia entre os anos de 1998 e 2007. Observou-se queda dos indicadores de morbidade das doenças respiratórias. Os principais fatores discutidos para esse declínio podem estar relacionados à melhoria dos serviços de saúde, principalmente no que diz respeito à prevenção destas afecções e à oferta de tratamento adequado. Estudos dessa natureza envolvendo a análise de número maior de variáveis são necessários para elucidar a correlação desses fatores e a tendência temporal da morbidade respiratória no município de Salvador.

\section{REFERÊNCIAS}

1. Toyoshima MTK, Ito GM, Gouveia N. Morbidade por doenças respiratórias em pacientes hospitalizados em São Paulo/SP. Rev Assoc Med Bras. 2005;51(4):20913. 
2. Mathias TAF, Soboll MLMS. Morbidade hospitalar em município da região Sul do Brasil em 1992. Rev Saude Publica. 1996;30(3):224-32.

3. Roseiro MNV, Takayanagui AMM. Morbidade por problemas respiratórios em Ribeirão Preto (SP) de 1995 a 2001, segundo indicadores ambientais, sociais e econômicos. Rev Paul Pediatria. 2006;24(2):163-70.

4. Carvalho LMT, Pereira EDB. Morbidade respiratória em crianças fumantes passivas. J Pneumol. 2002; 28(1):8-14.

5. Duchiade MP. Poluição do ar e doenças respiratórias: uma revisão. Cad Saude Publica. 1992;8(3):311-30.

6. Joad JP, Sekizawa S, Chen CY, Bonham AC. Air pollutants and cough. Pulm Pharmacol Ther. 2007; 20(4):347-54.

7. Eisner MD, Wang Y, Haight TJ, Balmes J, Hammond SK, Tager IB.Secondhand Smoke Exposure, Pulmonary Function, and Cardiovascular Mortality. Ann Epidemiol. 2007;17(5):364-73.

8. Freitas C, Bremner SA, Gouveia N, Pereira LAA, Saldiva PHN. Internações e óbitos e sua relação com a poluição atmosférica em São Paulo, 1993 a 1997. Rev Saude Publica. 2004;38(6):751-7.

9. Godoy DV, Zotto C, Bellicanta J, Weschenfelder RF, Nacif SB. Doenças respiratórias como causa de internações hospitalares de pacientes do Sistema Único de Saúde num serviço terciário de clínica médica na região nordeste do Rio Grande do Sul. J Pneumol. 2001;27(4):193-8.

10. Ministério da Saúde (BR). Datasus. [cited 2008 Nov 30]. Avaliabe from: http://www.datasus.gov.br.

11. Pereira MG. Variáveis relativas às pessoas. In: Pereira MG. Epidemiologia: teoria e prática. Rio de Janeiro: Guanabara Koogan; 1995. p.211.

12. Organização Mundial de Saúde. CID-10/ Organização Mundial de Saúde; tradução - Centro Colaborador da OMS para a Classificação de Doenças em Português. 3a ed. São Paulo: Editora da Universidade de São Paulo; 1996.

13. Toyoshima MTK, Ito GM, Gouveia N. Tendências temporais da morbidade e da mortalidade por doenças respiratórias na cidade de São Paulo. Rev Med. 2003; 82(1-4):67-77.
14. Silva BMP, Bispo DDC, Cardoso DNR, Rocha MTA, Ferreira MAF, Barretto NSA, Rêgo MAV. Tendência da morbimortalidade por pneumonia na região metropolitana de salvador - 1980 a 2004. Rev Baiana Saude Public. 2006;30(2):294-308.

15. Ferreira OS, Britto MCA. Pneumonia aguda - tema que todos devemos estudar. J Pediatria. 2003;79(6):478-9.

16. Teldeschi ALG, Sant'anna CC, Aires VLT. Prevalência de sintomas respiratórios e condições clínicas associadas à asma em escolares de 6 a 14 anos no Rio de Janeiro. Rev Assoc Med Bras. 2002;48(1):54-9.

17. Akinbami LJ, Schoendorf KC. Trends in Childhood Asthma: Prevalence, Health Care Utilization, and Mortality. Pediatrics. 2002;110(2 Pt 1):315-22.

18. Kolnaar BG, van Lier A, van den Bosch WJ, Folgering $\mathrm{H}$, van Herwaarden van den Hoogen HJ, van Weel C. Asthma in adolescents and young adults: relationship with early childhood respiratory morbidity. Br J Gen Pract. 1994;44(379):73-8.

19. Eisner MD, Katz PP, Yelin EH, Shiboski SC, Blanc PD. Risk factors for hospitalization among adults with asthma: the influence of sociodemographic factors and asthmaseverity. Respir Res. 2001;2(1):53-60.

20. Vargas MH, Díaz-Mejía GS, Furuya MEY, Salas J, Lugo A. Trends of Asthma in Mexico: An 11-Year Analysis in a Nationwide Institution. Chest. 2004; 125(6):1993-7.

21. Santos PM, D’Oliveira Júnior A, Noblat LACB, Machado AS, Noblat ACB, Cruz AA. Predictors of adherence to treatment in patients with severe asthma treated at a referral center in Bahia, Brazil. J Bras Pneumol. 2008 Dec; 34(12):995-1002.

22. Franco R, Santos AC, Nascimento HF, Souza-Machado C, Ponte E, Souza-Machodo A, et al. Cost-effectiveness analysis of a state funded programme for control of severe asthma. BMC Public Health. 2007;7(1):82.

23. Ponte E, Franco RA, Souza-Machado A, SouzaMachado C, Cruz AA. Impact that a program to control severe asthma has on the use of Unified Health System resources in Brazil. J Bras Pneumol. 2007 Feb;33(1):15-9.

24. Soto-Martinez M, Soto-Quiros M. Epidemiología del asma en Costa Rica. Rev méd Hosp Nac Niños (Costa Rica). 2004;39(1):42-53. 
25. Hamzaçebi H, Ünsal M, Kayhan S, Bilgin S, Ercan S. Prevalence of asthma and respiratory symptoms by age, gender and smoking behaviour in Samsun, North Anatolia Turkey. Tüberküloz ve Toraks Dergisi. 2006;54(4):322-9.

26. Felizola MLBM, Viegas CAA, Almeida M, Ferreira F, Santos MCA. Prevalence of bronchial asthma and related symptoms in schoolchildren in the Federal District of Brazil: correlations with socioeconomic levels. J Bras Pneumol. 2005;31(6):486-91.

27. Rothenbacher D, Arndt V, Fraisse E, Daniel U, Fliedner TM, Brenner H. Chronic respiratory disease morbidity in construction workers: patterns and prognostic significance for permanent disability and overall mortality. Eur Respir J. 1997;10(5):1093-9.

28. Lebrão ML. Determinantes da morbidade hospitalar em região do Estado de São Paulo (Brasil). Rev Saude Publica. 1999;33(1):55-63.
29. Mathias TAF, Soboll MLMS. Confiabilidade de diagnósticos nos formulários de autorização de internação hospitalar. Rev Saude Publica. 1998;32(6):526-32.

30. Veras CMT, Martins MAS. Confiabilidade dos dados nos formulários de Autorização de Internação Hospitalar (AIH). Cad Saude Publica. 1994; 10(3):33955.

\section{Endereço de correspondência:}

Jefferson Paixão Cardoso

Universidade Estadual do Sudoeste da Bahia

Departamento de Saúde

Avenida José Moreira Sobrinho S/N

Bairro: Jequiezinho

CEP: 45206-190 - Jequié - BA - Brasil

E:mail: jpcardoso@uesb.edu.br 